\title{
On asymptotically double lacunary statistical equivalent sequences in ideal context
}

\author{
Bipan Hazarika ${ }^{1 *}$ and Vijay Kumar ${ }^{2}$
}

${ }^{*}$ Correspondence:

bh_rgu@yahoo.co.in

${ }^{1}$ Department of Mathematics, Rajiv

Gandhi University, Rono Hills,

Doimukh, Arunachal Pradesh

791112, India

Full list of author information is

available at the end of the article

\begin{abstract}
An ideal $\mathcal{I}$ is a family of subsets of positive integers $\mathbb{N} \times \mathbb{N}$ which is closed under taking finite unions and subsets of its elements. In this paper, we present some definitions which are a natural combination of the definition of asymptotic equivalence, statistical convergence, lacunary statistical convergence, double sequences and an ideal. In addition, we also present asymptotically $\mathcal{I}$-equivalent double sequences and study some properties of this concept.
\end{abstract}

MSC: $40 \mathrm{~A} 35 ; 40 \mathrm{G} 15$

Keywords: asymptotic equivalence; statistical convergence; lacunary statistical convergence; ideal; ideal convergence; double sequence

\section{Introduction}

Pobyvancts [1] introduced the concept of asymptotically regular matrices which preserve the asymptotic equivalence of two nonnegative numbers sequences. Marouf [2] presented definitions for asymptotically equivalent and asymptotic regular matrices. Patterson [3] extended these concepts by presenting an asymptotically statistical equivalent analog of these definitions and natural regularity conditions for nonnegative summability matrices. Patterson and Savaş [4] introduced the concept of an asymptotically lacunary statistical equivalent sequences of real numbers.

The idea of statistical convergence was formerly given under the name 'almost convergence' by Zygmund in the first edition of his celebrated monograph published in Warsaw in 1935 [5]. The concept was formally introduced by Steinhaus [6] and Fast [7], and later, it was introduced by Schoenberg [8] and also independently by Buck [9]. A lot of developments have been made in this area after the works of Šalát [10] and Fridy [11]. Over the years and under different names, statistical convergence has been discussed in the theory of Fourier analysis, ergodic theory and number theory. Fridy and Orhan [12] introduced the concept of lacunary statistical convergence. Mursaleen and Mohiuddine [13] introduced the concept of lacunary statistical convergence with respect to the intuitionistic fuzzy normed space. Savaş and Patterson $[14,15]$ introduced the concept of lacunary statistical convergence for double sequences. Recently Mohiuddine et al. [16] introduced statistical convergence of double sequences in locally solid Riesz spaces. For details related to lacunary statistical convergence, we refer to [12, 17-24].

Kostyrko et al. [25] introduced the notion of $I$-convergence with the help of an admissible ideal, $I$ denotes the ideal of subsets of $\mathbb{N}$, which is a generalization of statistical convergence. Quite recently, Das et al. [26] unified these two approaches to introduce new

2013 Hazarika and Kumar: licensee Springer. This is an Open Access article distributed under the terms of the Creative Commons Attribution License (http://creativecommons.org/licenses/by/2.0), which permits unrestricted use, distribution, and reproduction in any medium, provided the original work is properly cited. 
concepts of $I$-statistical convergence, $I$-lacunary statistical convergence and investigated some of their consequences. The notion of lacunary ideal convergence of real sequences was introduced in [27, 28]. Hazarika [29, 30] introduced the lacunary ideal convergent sequences of fuzzy real numbers and studied some basic properties of this notion. Kumar and Sharma [31] studied asymptotically generalized statistical equivalent sequences using ideals. Recently Savas [32] and Savas and Gumus [33] studied ideal asymptotically lacunary statistical equivalent single sequences. For more applications of ideals, we refer to [34-47].

In this paper, we define asymptotically lacunary statistical equivalent double sequences using an ideal and establish some basic results regarding this notion.

\section{Definitions and preliminaries}

In this section, we recall some definitions and notations, which form the base for the present study.

A family of sets $I \subset P(\mathbb{N})$ (power sets of $\mathbb{N}$ ) is called an ideal if and only if for each $A, B \in I$, we have $A \cup B \in I$, and for each $A \in I$ and each $B \subset A$, we have $B \in I$. A non-empty family of sets $\mathcal{F} \subset P(\mathbb{N})$ is a filter on $\mathbb{N}$ if and only if $\phi \notin \mathcal{F}$, for each $A, B \in \mathcal{F}$, we have $A \cap B \in \mathcal{F}$ and each $A \in \mathcal{F}$ and each $B \supset A$, we have $B \in \mathcal{F}$. An ideal $I$ is called non-trivial ideal if $I \neq \phi$ and $\mathbb{N} \notin I$. Clearly, $I \subset P(\mathbb{N})$ is a non-trivial ideal if and only if $\mathcal{F}=\mathcal{F}(I)=\{\mathbb{N}-A: A \in I\}$ is a filter on $\mathbb{N}$. A non-trivial ideal $I \subset P(\mathbb{N})$ is called admissible if and only if $\{\{x\}: x \in \mathbb{N}\} \subset I$. A non-trivial ideal $I$ is maximal if there cannot exists any non-trivial ideal $J \neq I$ containing $I$ as a subset. Further details on ideals of $P(\mathbb{N})$ can be found in Kostyrko et al. [25]. Recall that a sequence $x=\left(x_{k}\right)$ of points in $\mathbb{R}$ is said to be $I$-convergent to a real number $\ell$ if $\left\{k \in \mathbb{N}:\left|x_{k}-\ell\right| \geq \varepsilon\right\} \in I$ for every $\varepsilon>0$ [25]. In this case, we write $I-\lim x_{k}=\ell$.

By a lacunary sequence $\theta=\left(k_{r}\right)$, where $k_{0}=0$, we mean an increasing sequence of nonnegative integers with $h_{r}:=k_{r}-k_{r-1} \rightarrow \infty$ as $r \rightarrow \infty$. The intervals determined by $\theta$ will be denoted by $J_{r}:=\left(k_{r-1}, k_{r}\right]$, and the ratio $\frac{k_{r}}{k_{r-1}}$ will be defined by $q_{r}$ (see [48]).

The notion of statistical convergence depends on the density (asymptotic or natural) of subsets of $\mathbb{N}$. A subset of $\mathbb{N}$ is said to have natural density $\delta(E)$ if

$$
\delta(E)=\lim _{n \rightarrow \infty} \frac{1}{n}|\{k \leq n: k \in E\}| \text { exists. }
$$

Definition 2.1 A real or complex number sequence $x=\left(x_{k}\right)$ is said to be statistically convergent to $L$ if for every $\varepsilon>0$,

$$
\lim _{n} \frac{1}{n}\left|\left\{k \leq n:\left|x_{k}-L\right| \geq \varepsilon\right\}\right|=0 .
$$

In this case, we write $S-\lim x=L$ or $x_{k} \rightarrow L(S)$, and $S$ denotes the set of all statistically convergent sequences.

Definition 2.2 [12] A sequence $x=\left(x_{k}\right)$ is said to be lacunary statistically convergent to the number $L$ if for every $\varepsilon>0$,

$$
\lim _{r \rightarrow \infty} \frac{1}{h_{r}}\left|\left\{k \in J_{r}:\left|x_{k}-L\right| \geq \varepsilon\right\}\right|=0 .
$$

Let $S_{\theta}$ denote the set of all lacunary statistically convergent sequences. If $\theta=\left(2^{r}\right)$, then $S_{\theta}$ is the same as $S$. 
Definition 2.3 [26] Let $I \subset P(\mathbb{N})$ be a non-trivial ideal. A sequence $\left(x_{k}\right)$ is $I$-statistically convergent to $L$ if for each $\varepsilon>0$ and $\delta>0$,

$$
\left\{n \in \mathbb{N}: \frac{1}{n}\left|\left\{k \leq n:\left|x_{k}-L\right| \geq \varepsilon\right\}\right| \geq \delta\right\} \in I .
$$

In this case, we write $I(S)-\lim x_{k}=L$.

Definition 2.4 [26] Let $I \subset P(\mathbb{N})$ be a non-trivial ideal. A sequence $\left(x_{k}\right)$ is said to be $I$ lacunary statistically convergent to $L$ if for each $\varepsilon>0$ and $\delta>0$,

$$
\left\{r \in \mathbb{N}: \frac{1}{h_{r}}\left|\left\{k \in J_{r}:\left|x_{k}-L\right| \geq \varepsilon\right\}\right| \geq \delta\right\} \in I .
$$

In this case, we write $I\left(S_{\theta}\right)-\lim x_{k}=L$. If $\theta=\left(2^{r}\right)$, then $I\left(S_{\theta}\right)$ is the same as $I(S)$.

Definition 2.5 [2] Two nonnegative sequences $x=\left(x_{k}\right)$ and $y=\left(y_{k}\right)$ are said to be asymptotically equivalent if

$$
\lim _{k} \frac{x_{k}}{y_{k}}=1 \text {, }
$$

denoted by $x \sim y$.

Definition 2.6 [3] Two nonnegative sequences $x=\left(x_{k}\right)$ and $y=\left(y_{k}\right)$ are said to be asymptotically statistical equivalent of multiple $L$ provided that for every $\varepsilon>0$,

$$
\lim _{n} \frac{1}{n}\left|\left\{k \leq n:\left|\frac{x_{k}}{y_{k}}-L\right| \geq \varepsilon\right\}\right|=0
$$

denoted by $x \sim S^{L} y$ and simply asymptotically statistical equivalent if $L=1$.

Patterson and Savas [4] defined the asymptotically lacunary statistical equivalent sequences as follows.

Definition 2.7 Two nonnegative sequences $x=\left(x_{k}\right)$ and $y=\left(y_{k}\right)$ are said to be asymptotically lacunary statistical equivalent of multiple $L$ provided that for every $\varepsilon>0$,

$$
\lim _{r} \frac{1}{h_{r}}\left|\left\{k \in J_{r}:\left|\frac{x_{k}}{y_{k}}-L\right| \geq \varepsilon\right\}\right|=0
$$

denoted by $x \sim S_{\theta}^{L} y$ and simply asymptotically lacunary statistical equivalent if $L=1$. If we take $\theta=\left(2^{r}\right)$, then we get Definition 2.6.

By the convergence of a double sequence, we mean the convergence in Pringsheim's sense [49]. A double sequence $x=\left(x_{k, l}\right)$ has a Pringsheim limit $L$ (denoted by $P-\lim x=L$ ) provided that given an $\varepsilon>0$, there exists an $n \in \mathbb{N}$ such that $\left|x_{k, l}-L\right|<\varepsilon$, whenever $k, l>n$. We describe such an $x=\left(x_{k, l}\right)$ more briefly as ' $P$-convergent'. We denote the space of all $P$-convergent sequences by $c^{2}$. The double sequence $x=\left(x_{k, l}\right)$ is bounded if there exists 
a positive number $M$ such that $\left|x_{k, l}\right|<M$ for all $k$ and $l$. We denote all bounded double sequences by $l_{\infty}^{2}$.

Let $K \subset \mathbb{N} \times \mathbb{N}$ and $K(m, n)$ denote the number of $(i, j)$ in $K$ such that $i \leq m$ and $j \leq n$ (see [50]). Then the lower natural density of $K$ is defined by $\underline{\delta}_{2}(K)=\liminf \inf _{m, n \rightarrow \infty} \frac{K(m, n)}{m n}$. In case the sequence $\left(\frac{K(m, n)}{m n}\right)$ has a limit in Pringsheim's sense, then we say that $K$ has a double natural density and is defined by $P-\lim _{m, n \rightarrow \infty} \frac{K(m, n)}{m n}=\delta_{2}(K)$.

For example, let $K=\left\{\left(i^{2}, j^{2}\right): i, j \in \mathbb{N}\right\}$. Then

$$
\delta_{2}(K)=P-\lim _{m, n \rightarrow \infty} \frac{K(m, n)}{m n} \leq P-\lim _{m, n \rightarrow \infty} \frac{\sqrt{m} \sqrt{n}}{m n}=0,
$$

i.e., the set $K$ has double natural density zero, while the set $\{(i, 3 j): i, j \in \mathbb{N}\}$ has double natural density $\frac{1}{3}$.

Definition 2.8 [50] A real double sequence $x=\left(x_{k, l}\right)$ is said to be P-statistically convergent to $\ell$ provided that for each $\varepsilon>0$,

$$
P-\lim _{m n} \frac{1}{m n} \mid\left\{(k, l): k<m \text { and } l<n,\left|x_{k, l}-\ell\right| \geq \varepsilon\right\} \mid=0 .
$$

We denote the set of all statistical convergent double sequences by $\mathcal{S}$.

The double sequence $\bar{\theta}=\theta_{r, s}=\left\{\left(k_{r}, l_{s}\right)\right\}$ is called double lacunary sequence if there exist two increasing sequences of integers such that (see [15])

$$
k_{o}=0, \quad h_{r}=k_{r}-k_{r-1} \rightarrow \infty \quad \text { as } r \rightarrow \infty
$$

and

$$
l_{o}=0, \quad \overline{h_{s}}=l_{s}-l_{s-1} \rightarrow \infty \quad \text { as } s \rightarrow \infty .
$$

Notations: $k_{r, s}=k_{r} l_{s}, h_{r, s}=h_{r} \overline{h_{s}}$ and $\theta_{r, s}$ is determined by

$$
\begin{aligned}
& J_{r, s}=\left\{(k, l): k_{r-1}<k \leq k_{r} \text { and } l_{s-1}<l \leq l_{s}\right\}, \\
& q_{r}=\frac{k_{r}}{k_{r-1}}, \quad \overline{q_{s}}=\frac{l_{s}}{l_{s-1}} \quad \text { and } \quad q_{r, s}=q_{r} \overline{q_{s}} .
\end{aligned}
$$

Definition 2.9 A double sequence $\left(x_{k, l}\right)$ is said to be double lacunary convergent to $L$ if

$$
P-\lim _{r, s} \frac{1}{h_{r, s}} \sum_{(k, l) \in J_{r, s}} x_{k, l}=L
$$

In this case, we write $\bar{\theta}-\lim _{k, l} x_{k, l}=L$. We denote $N_{\bar{\theta}}$ the set of all double lacunary convergent sequences.

Definition 2.10 [51] Let $\mathcal{I} \subset P(\mathbb{N} \times \mathbb{N})$ be a non-trivial ideal. A double sequence $\left(x_{k, l}\right)$ is said to be $\mathcal{I}$-convergent to $L$ if for each $\varepsilon>0$,

$$
\left\{(k, l) \in \mathbb{N} \times \mathbb{N}:\left|x_{k, l}-L\right| \geq \varepsilon\right\} \in \mathcal{I} .
$$

In this case, we write $\mathcal{I}-\lim x_{k, l}=L$. 
Throughout the paper, we denote $\mathcal{I}$ as admissible ideal of subsets of $\mathbb{N} \times \mathbb{N}$, unless otherwise stated.

\section{Asymptotically lacunary statistical equivalent double sequences using ideals}

In this section, we define asymptotically $\mathcal{I}$-equivalent, asymptotically $\mathcal{I}$-statistical equivalent, asymptotically $\mathcal{I}$-lacunary statistical equivalent and asymptotically lacunary $\mathcal{I}$ equivalent double sequences and obtain some analogous results from these new definitions point of views.

Definition 3.1 Let $\mathcal{I} \subset P(\mathbb{N} \times \mathbb{N})$ be a non-trivial ideal. A double sequence $\left(x_{k, l}\right)$ is said to be $\mathcal{I}$-statistically convergent to $L$ if for each $\varepsilon>0$ and $\delta>0$,

$$
\left\{(m, n) \in \mathbb{N} \times \mathbb{N}: \frac{1}{m n}\left|\left\{k \leq m ; l \leq n:\left|x_{k, l}-L\right| \geq \varepsilon\right\}\right| \geq \delta\right\} \in \mathcal{I} .
$$

In this case, we write $\mathcal{I}(\mathcal{S})-\lim x_{k, l}=L$.

Definition 3.2 Let $\mathcal{I} \subset P(\mathbb{N} \times \mathbb{N})$ be a non-trivial ideal. A double sequence $\left(x_{k, l}\right)$ is said to be $\mathcal{I}$-lacunary statistically convergent to $L$ if for each $\varepsilon>0$ and $\delta>0$,

$$
\left\{(r, s) \in \mathbb{N} \times \mathbb{N}: \frac{1}{h_{r, s}}\left|\left\{(k, l) \in J_{r, s}:\left|x_{k, l}-L\right| \geq \varepsilon\right\}\right| \geq \delta\right\} \in \mathcal{I} .
$$

In this case, we write $\mathcal{I}\left(\mathcal{S}_{\bar{\theta}}\right)-\lim x_{k, l}=L$.

Definition 3.3 Two nonnegative double sequences $x=\left(x_{k, l}\right)$ and $y=\left(y_{k, l}\right)$ are said to be P-asymptotically equivalent if

$$
P-\lim _{k, l} \frac{x_{k, l}}{y_{k, l}}=1,
$$

denoted by $x \sim^{P} y$.

Definition 3.4 Two nonnegative double sequences $x=\left(x_{k, l}\right)$ and $y=\left(y_{k, l}\right)$ are said to be asymptotically statistical equivalent of multiple $L$ provided that for every $\varepsilon>0$

$$
P-\lim _{m, n} \frac{1}{m n}\left|\left\{k \leq m, l \leq n:\left|\frac{x_{k, l}}{y_{k, l}}-L\right| \geq \varepsilon\right\}\right|=0
$$

denoted by $x \sim \mathcal{S}^{L} y$ and simply asymptotically statistical equivalent if $L=1$.

Definition 3.5 Two nonnegative double sequences $x=\left(x_{k, l}\right)$ and $y=\left(y_{k, l}\right)$ are said to be asymptotically lacunary statistical equivalent of multiple $L$ provided that for every $\varepsilon>0$,

$$
P-\lim _{r, s} \frac{1}{h_{r, s}}\left|\left\{(k, l) \in J_{r, s}:\left|\frac{x_{k, l}}{y_{k, l}}-L\right| \geq \varepsilon\right\}\right|=0
$$

denoted by $x \sim \mathcal{S}_{\bar{\theta}}^{L} y$ and simply asymptotically lacunary statistical equivalent if $L=1$. If we take $\bar{\theta}=\left(2^{r}, 2^{s}\right)$, then we get Definition 3.4. 
Definition 3.6 Two non-negative double sequences $\left(x_{k, l}\right)$ and $\left(y_{k, l}\right)$ are said to be asymptotically $\mathcal{I}$-equivalent of multiple $L$ provided that for every $\varepsilon>0$,

$$
\left\{(k, l) \in \mathbb{N} \times \mathbb{N}:\left|\frac{x_{k, l}}{y_{k, l}}-L\right| \geq \varepsilon\right\} \in \mathcal{I},
$$

denoted by $\left(x_{k, l}\right) \sim \mathcal{I}^{L}\left(y_{k, l}\right)$ and simply asymptotically $\mathcal{I}$-equivalent if $L=1$.

Lemma 3.1 Let $\mathcal{I} \subset P(\mathbb{N} \times \mathbb{N})$ be an admissible ideal. Let $\left(x_{k, l}\right),\left(y_{k, l}\right)$ be two double sequences and $\left(x_{k, l}\right),\left(y_{k, l}\right) \in \ell_{\infty}^{2}$ with $\mathcal{I}-\lim _{k, l} x_{k, l}=0=\mathcal{I}-\lim _{k, l} y_{k, l}$ such that $\left(x_{k, l}\right) \sim \mathcal{I}^{L}\left(y_{k, l}\right)$. Then there exists a sequence $\left(z_{k, l}\right) \in \ell_{\infty}^{2}$ with $\mathcal{I}-\lim _{k, l} z_{k, l}=0$ such that $\left(x_{k, l}\right) \sim \mathcal{I}^{L}\left(z_{k, l}\right) \sim \mathcal{I}^{L}$ $\left(y_{k, l}\right)$.

Definition 3.7 Two non-negative double sequences $\left(x_{k, l}\right)$ and $\left(y_{k, l}\right)$ are said to be asymptotically $\mathcal{I}$-statistically equivalent of multiple $L$ provided that for every $\varepsilon>0$ and for every $\delta>0$,

$$
\left\{(m, n) \in \mathbb{N} \times \mathbb{N}: \frac{1}{m n}\left|\left\{k \leq m, l \leq n:\left|\frac{x_{k, l}}{y_{k, l}}-L\right| \geq \varepsilon\right\}\right| \geq \delta\right\} \in \mathcal{I},
$$

denoted by $\left(x_{k, l}\right) \sim \mathcal{I}(\mathcal{S})^{L}\left(y_{k, l}\right)$ and simply asymptotically $\mathcal{I}$-statistical equivalent if $L=1$.

Definition 3.8 Two non-negative double sequences $\left(x_{k, l}\right)$ and $\left(y_{k, l}\right)$ are said to be Cesaro asymptotically $\mathcal{I}$-equivalent (or $\mathcal{I}\left(\sigma_{1}\right)$-equivalent) of multiple $L$ provided that for every $\delta>0$,

$$
\left\{(m, n) \in \mathbb{N} \times \mathbb{N}:\left|\frac{1}{m n} \sum_{k, l=1}^{m, n} \frac{x_{k, l}}{y_{k, l}}-L\right| \geq \delta\right\} \in \mathcal{I}
$$

denoted by $\left(x_{k, l}\right) \sim \mathcal{I}\left(\sigma_{1}\right)^{L}\left(y_{k, l}\right)$ and simply asymptotically $\mathcal{I}\left(\sigma_{1}\right)$-equivalent if $L=1$.

Definition 3.9 Two non-negative double sequences $\left(x_{k, l}\right)$ and $\left(y_{k, l}\right)$ are said to be strongly Cesaro asymptotically $\mathcal{I}$-equivalent (or $\mathcal{I}\left(\left|\sigma_{1}\right|\right)$-equivalent) of multiple $L$ provided that for every $\delta>0$,

$$
\left\{(m, n) \in \mathbb{N} \times \mathbb{N}: \frac{1}{m n} \sum_{k, l=1}^{m, n}\left|\frac{x_{k, l}}{y_{k, l}}-L\right| \geq \delta\right\} \in \mathcal{I}
$$

denoted by $\left(x_{k, l}\right) \sim^{\mathcal{I}\left(\left|\sigma_{1}\right|\right)^{L}}\left(y_{k, l}\right)$ and simply strongly Cesaro asymptotically $\mathcal{I}\left(\left|\sigma_{1}\right|\right)$-equivalent if $L=1$.

Definition 3.10 Two non-negative double sequences $\left(x_{k, l}\right)$ and $\left(y_{k, l}\right)$ are said to be strongly asymptotically lacunary equivalent of multiple $L$ provided that

$$
P-\lim _{r, s} \frac{1}{h_{r, s}} \sum_{(k, l) \in J_{r, s}}\left|\frac{x_{k, l}}{y_{k, l}}-L\right|=0
$$

denoted by $\left(x_{k, l}\right) \sim \sim^{\left[N_{\bar{\theta}}^{-}\right]^{L}}\left(y_{k, l}\right)$ and simply strongly asymptotically lacunary equivalent if $L=1$. 
Definition 3.11 Two non-negative double sequences $\left(x_{k, l}\right)$ and $\left(y_{k, l}\right)$ are said to be asymptotically $\mathcal{I}$-lacunary equivalent (or $\mathcal{I}\left(N_{\bar{\theta}}\right)$-equivalent) of multiple $L$ provided that for every $\delta>0$,

$$
\left\{(r, s) \in \mathbb{N} \times \mathbb{N}: \frac{1}{h_{r, s}} \sum_{(k, l) \in I_{r, s}}\left|\frac{x_{k, l}}{y_{k, l}}-L\right| \geq \delta\right\} \in \mathcal{I}
$$

denoted by $\left(x_{k, l}\right) \sim^{\mathcal{I}\left(N_{\bar{\theta}}\right)^{L}}\left(y_{k, l}\right)$ and simply asymptotically $\mathcal{I}\left(N_{\bar{\theta}}\right)$-equivalent if $L=1$.

Definition 3.12 Two non-negative double sequences $\left(x_{k, l}\right)$ and $\left(y_{k, l}\right)$ are said to be asymptotically $\mathcal{I}$-lacunary statistically equivalent (or $\mathcal{I}\left(\mathcal{S}_{\bar{\theta}}\right)$-equivalent) of multiple $L$ provided that for every $\varepsilon>0$, for every $\delta>0$,

$$
\left\{(r, s) \in \mathbb{N} \times \mathbb{N}: \frac{1}{h_{r, s}}\left|\left\{(k, l) \in J_{r, s}:\left|\frac{x_{k, l}}{y_{k, l}}-L\right| \geq \varepsilon\right\}\right| \geq \delta\right\} \in \mathcal{I}
$$

denoted by $\left(x_{k, l}\right) \sim^{\mathcal{I}\left(\mathcal{S}_{\bar{\theta}}\right)^{L}}\left(y_{k, l}\right)$ and simply asymptotically $\mathcal{I}\left(\mathcal{S}_{\bar{\theta}}\right)$-equivalent if $L=1$.

Theorem 3.1 Let $\mathcal{I} \subset P(\mathbb{N} \times \mathbb{N})$ be a non-trivial ideal. Let $\bar{\theta}=\left\{\left(k_{r}, l_{s}\right)\right\}$ be a double lacunary sequence. If $\left(x_{k, l}\right),\left(y_{k, l}\right) \in \ell_{\infty}^{2}$ and $\left(x_{k, l}\right) \sim^{\mathcal{I}(\mathcal{S})^{L}}\left(y_{k, l}\right)$. Then $\left(x_{k, l}\right) \sim \mathcal{I}\left(\sigma_{1}\right)^{L}\left(y_{k, l}\right)$.

Proof (a) Suppose that $\left(x_{k, l}\right),\left(y_{k, l}\right) \in \ell_{\infty}^{2}$ and $\left(x_{k, l}\right) \sim \mathcal{I}(\mathcal{S})^{L}\left(y_{k, l}\right)$. Then we can assume that

$$
\left|\frac{x_{k, l}}{y_{k, l}}-L\right| \leq M \quad \text { for almost all } k, l \text {. }
$$

Let $\varepsilon>0$. Then we have

$$
\begin{aligned}
\left|\frac{1}{m n} \sum_{k, l=1}^{m, n}\left(\frac{x_{k, l}}{y_{k, l}}-L\right)\right| & \leq \frac{1}{m n} \sum_{k, l=1}^{m, n}\left|\frac{x_{k, l}}{y_{k, l}}-L\right| \\
& \leq \frac{1}{m n} \sum_{\substack{k, l ; \\
\left|\frac{x_{k, l}}{y_{k, l}}-L\right| \geq \varepsilon}}\left|\frac{x_{k, l}}{y_{k, l}}-L\right|+\frac{1}{m n} \sum_{\substack{k, l ; \\
\left|\frac{k_{k, l}}{y_{k, l}}-L\right|<\varepsilon}}\left|\frac{x_{k, l}}{y_{k, l}}-L\right| \\
& \leq M \cdot \frac{1}{m n}\left|\left\{k \leq m ; l \leq n:\left|\frac{x_{k, l}}{y_{k, l}}-L\right| \geq \varepsilon\right\}\right|+\frac{1}{m n} \cdot m n \cdot \varepsilon .
\end{aligned}
$$

Consequently, if $\delta>\varepsilon>0, \delta$ and $\varepsilon$ are independent, put $\delta_{1}=\delta-\varepsilon>0$, we have

$$
\begin{aligned}
& \left\{(m, n) \in \mathbb{N} \times \mathbb{N}:\left|\frac{1}{m n} \sum_{k, l=1}^{m, n}\left(\frac{x_{k, l}}{y_{k, l}}-L\right)\right| \geq \delta\right\} \\
& \quad \subseteq\left\{(m, n) \in \mathbb{N} \times \mathbb{N}: \frac{1}{m n}\left|\left\{k \leq m ; l \leq n:\left|\frac{x_{k, l}}{y_{k, l}}-L\right| \geq \varepsilon\right\}\right| \geq \frac{\delta_{1}}{M}\right\} \in \mathcal{I} .
\end{aligned}
$$

This shows that $\left(x_{k, l}\right) \sim^{\mathcal{I}\left(\sigma_{1}\right)^{L}}\left(y_{k, l}\right)$.

Corollary 3.1 Let $\mathcal{I} \subset P(\mathbb{N} \times \mathbb{N})$ be a non-trivial ideal. If $\left(x_{k, l}\right),\left(y_{k, l}\right) \in \ell_{\infty}^{2}$ and $\left(x_{k, l}\right) \sim \mathcal{I}(\mathcal{S})^{L}$ $\left(y_{k, l}\right)$. Then $\left(x_{k, l}\right) \sim \mathcal{I}\left(\left|\sigma_{1}\right|\right)^{L}\left(y_{k, l}\right)$. 
Theorem 3.2 Let $\mathcal{I} \subset P(\mathbb{N} \times \mathbb{N})$ be a non-trivial ideal. Let $\bar{\theta}=\left\{\left(k_{r}, l_{s}\right)\right\}$ be a double lacunary sequence. Then

(a) $\left(x_{k, l}\right) \sim^{\mathcal{I}\left(N_{\bar{\theta}}\right)^{L}}\left(y_{k, l}\right) \Rightarrow\left(x_{k, l}\right) \sim^{\mathcal{I}\left(\mathcal{S}_{\bar{\theta}}\right)^{L}}\left(y_{k, l}\right)$.

(b) $\mathcal{I}\left(N_{\bar{\theta}}\right)^{L}$ is a proper subset of $\mathcal{I}\left(\mathcal{S}_{\bar{\theta}}\right)^{L}$.

(c) Let $\left(x_{k, l}\right),\left(y_{k, l}\right) \in \ell_{\infty}^{2}$ and $\left(x_{k, l}\right) \sim^{\mathcal{I}\left(\mathcal{S}_{\bar{\theta}}\right)^{L}}\left(y_{k, l}\right)$, then $\left(x_{k, l}\right) \sim^{\mathcal{I}\left(N_{\bar{\theta}}\right)^{L}}\left(y_{k, l}\right)$.

(d) $\mathcal{I}\left(\mathcal{S}_{\bar{\theta}}\right)^{L} \cap \ell_{\infty}^{2}=\mathcal{I}\left(N_{\bar{\theta}}\right)^{L} \cap \ell_{\infty}^{2}$.

Proof (a) Let $\varepsilon>0$ and $\left(x_{k, l}\right) \sim^{\mathcal{I}\left(N_{\bar{\theta}}\right)^{L}}\left(y_{k, l}\right)$. Then we can write

$$
\begin{aligned}
\sum_{(k, l) \in J_{r, s}}\left|\frac{x_{k, l}}{y_{k, l}}-L\right| & \geq \sum_{\substack{(k, l) \in J_{r, s} \\
\left|\frac{x_{k, l}}{y_{k, l}}-L\right| \geq \varepsilon}}\left|\frac{x_{k, l}}{y_{k, l}}-L\right| \\
& \geq \varepsilon\left|\left\{(k, l) \in J_{r, s}:\left|\frac{x_{k, l}}{y_{k, l}}-L\right| \geq \varepsilon\right\}\right| \\
\Rightarrow \quad \frac{1}{\varepsilon \cdot h_{r, s}} & \sum_{(k, l) \in I_{r, s}}\left|\frac{x_{k, l}}{y_{k, l}}-L\right| \geq \frac{1}{h_{r, s}}\left|\left\{(k, l) \in J_{r, s}:\left|\frac{x_{k, l}}{y_{k, l}}-L\right| \geq \varepsilon\right\}\right| .
\end{aligned}
$$

Thus, for any $\delta>0$,

$$
\frac{1}{h_{r, s}}\left|\left\{(k, l) \in J_{r, s}:\left|\frac{x_{k, l}}{y_{k, l}}-L\right| \geq \varepsilon\right\}\right| \geq \delta
$$

implies that

$$
\frac{1}{h_{r, s}} \sum_{(k, l) \in J_{r, s}}\left|\frac{x_{k, l}}{y_{k, l}}-L\right| \geq \varepsilon \delta
$$

Therefore, we have

$$
\begin{gathered}
\left\{(r, s) \in \mathbb{N} \times \mathbb{N}: \frac{1}{h_{r, s}}\left|\left\{(k, l) \in J_{r, s}:\left|\frac{x_{k, l}}{y_{k, l}}-L\right| \geq \varepsilon\right\}\right| \geq \delta\right\} \\
\subset\left\{(r, s) \in \mathbb{N} \times \mathbb{N}: \frac{1}{h_{r, s}} \sum_{(k, l) \in J_{r, s}}\left|\frac{x_{k, l}}{y_{k, l}}-L\right| \geq \varepsilon \delta\right\} .
\end{gathered}
$$

Since $\left(x_{k, l}\right) \sim^{\mathcal{I}\left(N_{\bar{\theta}}\right)^{L}}\left(y_{k, l}\right)$, so that

$$
\left\{(r, s) \in \mathbb{N} \times \mathbb{N}: \frac{1}{h_{r, s}} \sum_{(k, l) \in J_{r, s}}\left|\frac{x_{k, l}}{y_{k, l}}-L\right| \geq \varepsilon \delta\right\} \in \mathcal{I},
$$

which implies that

$$
\left\{(r, s) \in \mathbb{N} \times \mathbb{N}: \frac{1}{h_{r, s}}\left|\left\{(k, l) \in J_{r, s}:\left|\frac{x_{k, l}}{y_{k, l}}-L\right| \geq \varepsilon\right\}\right| \geq \delta\right\} \in \mathcal{I} .
$$

This shows that $\left(x_{k, l}\right) \sim^{\mathcal{I}\left(\mathcal{S}_{\bar{\theta}}\right)^{L}}\left(y_{k, l}\right)$. 
(b) Suppose that $\mathcal{I}\left(N_{\bar{\theta}}\right)^{L} \subset \mathcal{I}\left(\mathcal{S}_{\bar{\theta}}\right)^{L}$. Let $\left(x_{k, l}\right)$ and $\left(y_{k, l}\right)$ be two sequences defined as follows:

$$
x_{k, l}= \begin{cases}k l, & \text { if } k_{r-1}<k \leq k_{r-1}+\left[\sqrt{h_{r}}\right], l_{s-1}<l \leq l_{s-1}+\left[\sqrt{h_{s}}\right], r, s=1,2,3, \ldots ; \\ 0, & \text { otherwise }\end{cases}
$$

and

$y_{k, l}=1 \quad$ for all $k, l \in \mathbb{N}$.

It is clear that $\left(x_{k, l}\right) \notin \ell_{\infty}^{2}$, and for $\varepsilon>0$,

$$
\frac{1}{h_{r, s}}\left|\left\{(k, l) \in J_{r, s}:\left|\frac{x_{k, l}}{y_{k, l}}-1\right| \geq \varepsilon\right\}\right| \leq \frac{\left[\sqrt{h_{r, s}}\right]}{h_{r, s}} \quad \text { and } \quad \frac{\left[\sqrt{h_{r, s}}\right]}{h_{r, s}} \rightarrow 0 \quad \text { as } r, s \rightarrow \infty \text {. }
$$

This implies that

$$
\begin{aligned}
& \left\{(r, s) \in \mathbb{N} \times \mathbb{N}: \frac{1}{h_{r, s}}\left|\left\{(k, l) \in J_{r, s}:\left|\frac{x_{k, l}}{y_{k, l}}-1\right| \geq \varepsilon\right\}\right| \geq \delta\right\} \\
& \subseteq\left\{(r, s) \in \mathbb{N} \times \mathbb{N}: \frac{\left[\sqrt{h_{r, s}}\right]}{h_{r, s}} \geq \delta\right\} .
\end{aligned}
$$

By virtue of last part of (3.1), the set on the right side is a finite set, and so it belongs to $\mathcal{I}$. Consequently, we have

$$
\left\{(r, s) \in \mathbb{N} \times \mathbb{N}: \frac{1}{h_{r, s}}\left|\left\{(k, l) \in J_{r, s}:\left|\frac{x_{k, l}}{y_{k, l}}-1\right| \geq \varepsilon\right\}\right| \geq \delta\right\} \in \mathcal{I} .
$$

Therefore, $\left(x_{k, l}\right) \sim \mathcal{I}\left(\mathcal{S}_{\bar{\theta}}\right)^{1}\left(y_{k, l}\right)$.

On the other hand, we shall show that $\left(x_{k, l}\right) \sim \mathcal{I}\left(N_{\bar{\theta}}\right)^{1}\left(y_{k, l}\right)$ is not satisfied. Suppose that $\left(x_{k, l}\right) \sim \mathcal{I}\left(N_{\bar{\theta}}\right)^{1}\left(y_{k, l}\right)$. Then for every $\delta>0$, we have

$$
\left\{(r, l) \in \mathbb{N} \times \mathbb{N}: \frac{1}{h_{r, s}} \sum_{(k, l) \in J_{r, s}}\left|\frac{x_{k, l}}{y_{k, l}}-1\right| \geq \delta\right\} \in \mathcal{I}
$$

Now,

$$
\lim _{r, s} \frac{1}{h_{r, s}} \sum_{(k, l) \in J_{r, s}}\left|\frac{x_{k, l}}{y_{k, l}}-1\right|=\frac{1}{h_{r, s}}\left(\frac{\left[\sqrt{h_{r, s}}\right]\left(\left[\sqrt{h_{r, s}}\right]-1\right)}{2}\right) \rightarrow \frac{1}{2} \quad \text { as } r, s \rightarrow \infty .
$$

It follows for the particular choice $\delta=\frac{1}{4}$ that

$$
\begin{aligned}
& \left\{(r, s) \in \mathbb{N} \times \mathbb{N}: \frac{1}{h_{r, s}} \sum_{(k, l) \in J_{r, s}}\left|\frac{x_{k, l}}{y_{k, l}}-1\right| \geq \frac{1}{4}\right\} \\
& \quad=\left\{(r, s) \in \mathbb{N} \times \mathbb{N}:\left(\frac{\left[\sqrt{h_{r, s}}\right]\left(\left[\sqrt{h_{r, s}}\right]-1\right)}{h_{r, s}}\right) \geq \frac{1}{2}\right\} \\
& \quad=\{(m, n),(m+1, n+1),(m+2, n+2), \ldots\}
\end{aligned}
$$


for some $m, n \in \mathbb{N}$ which belongs to $\mathcal{F}$ as $\mathcal{I}$ is admissible. This contradicts (3.2) for the choice $\delta=\frac{1}{4}$. Therefore, $\left(x_{k, l}\right) \nsim^{\mathcal{I}\left(N_{\bar{\theta}}\right)^{1}}\left(y_{k, l}\right)$.

(c) Suppose that $\left(x_{k, l}\right) \sim \mathcal{I}\left(\mathcal{S}_{\bar{\theta}}\right)^{L}\left(y_{k, l}\right)$ and $\left(x_{k, l}\right),\left(y_{k, l}\right) \in \ell_{\infty}^{2}$. We assume that $\left|\frac{x_{k, l}}{y_{k, l}}-L\right| \leq M$ and for all $k, l \in \mathbb{N}$. Given $\varepsilon>0$, we get

$$
\begin{aligned}
\frac{1}{h_{r, s}} \sum_{(k, l) \in J_{r, s}}\left|\frac{x_{k, l}}{y_{k, l}}-L\right| & =\frac{1}{h_{r, s}} \sum_{\substack{(k, l) \in I_{r, s} \\
\left|\frac{x_{k, l}}{y_{k, l}}-L\right| \geq \varepsilon}}\left|\frac{x_{k, l}}{y_{k, l}}-L\right|+\frac{1}{h_{r, s}} \sum_{\substack{(k, l) \in I_{r, s} \\
\left|\frac{x_{k, l}}{y_{k, l}}-L\right|<\varepsilon}}\left|\frac{x_{k, l}}{y_{k, l}}-L\right| \\
& \leq \frac{M}{h_{r, s}}\left|\left\{(k, l) \in J_{r, s}:\left|\frac{x_{k, l}}{y_{k, l}}-L\right| \geq \varepsilon\right\}\right|+\varepsilon .
\end{aligned}
$$

If we put

$$
A(\varepsilon)=\left\{(r, s) \in \mathbb{N} \times \mathbb{N}: \frac{1}{h_{r, s}} \sum_{(k, l) \in J_{r, s}}\left|\frac{x_{k, l}}{y_{k, l}}-L\right| \geq \varepsilon\right\}
$$

and

$$
B\left(\varepsilon_{1}\right)=\left\{(r, s) \in \mathbb{N} \times \mathbb{N}: \frac{1}{h_{r, s}}\left|\left\{(k, l) \in J_{r, s}:\left|\frac{x_{k, l}}{y_{k, l}}-L\right| \geq \varepsilon\right\}\right| \geq \frac{\varepsilon_{1}}{M}\right\},
$$

where $\varepsilon_{1}=\delta-\varepsilon>0$, ( $\delta$ and $\varepsilon$ are independent), then we have $A(\varepsilon) \subset B\left(\varepsilon_{1}\right)$, and so $A(\varepsilon) \in \mathcal{I}$. This shows that $\left(x_{k, l}\right) \sim \mathcal{I}\left(N_{\bar{\theta}}\right)^{L}\left(y_{k, l}\right)$.

(d) It follows from (a), (b) and (c).

Theorem 3.3 Let $\mathcal{I} \subset P(\mathbb{N} \times \mathbb{N})$ be an admissible ideal. Suppose that for given $\delta>0$ and every $\varepsilon>0$ such that

$$
\left\{(m, n) \in \mathbb{N} \times \mathbb{N}: \frac{1}{m n}\left|\left\{0 \leq k \leq m-1 ; 0 \leq l \leq n-1:\left|\frac{x_{k, l}}{y_{k, l}}-L\right| \geq \varepsilon\right\}\right|<\delta\right\} \in \mathcal{F},
$$

then $\left(x_{k, l}\right) \sim^{\mathcal{I}(\mathcal{S})^{L}}\left(y_{k, l}\right)$.

Proof Let $\delta>0$ be given. For every $\varepsilon>0$, choose $m_{1}, n_{1}$ such that

$$
\begin{aligned}
& \frac{1}{m n}\left|\left\{0 \leq k \leq m-1 ; 0 \leq l \leq n-1:\left|\frac{x_{k, l}}{y_{k, l}}-L\right| \geq \varepsilon\right\}\right| \\
& <\frac{\delta}{2}, \quad \text { for all } m \geq m_{1}, n \geq n_{1} .
\end{aligned}
$$

It is sufficient to show that there exists $m_{2}, n_{2}$ such that for $m \geq m_{2}, n \geq n_{2}$,

$$
\frac{1}{m n}\left|\left\{0 \leq k \leq m-1 ; 0 \leq l \leq n-1:\left|\frac{x_{k, l}}{y_{k, l}}-L\right| \geq \varepsilon\right\}\right|<\frac{\delta}{2} .
$$

Let $m_{0}=\max \left\{m_{1}, m_{2}\right\} ; n_{0}=\max \left\{n_{1}, n_{2}\right\}$. The relation (3.3) will be true for $m>m_{0}, n>n_{0}$. If $s_{0}, t_{0}$ chosen fixed, then we get

$$
\left|\left\{0 \leq k \leq s_{0}-1 ; 0 \leq l \leq t_{0}-1:\left|\frac{x_{k, l}}{y_{k, l}}-L\right| \geq \varepsilon\right\}\right|=M .
$$


Now, for $m>s_{0}, n>t_{0}$, we have

$$
\begin{aligned}
\frac{1}{m n}\left|\left\{0 \leq k \leq m-1 ; 0 \leq l \leq n-1:\left|\frac{x_{k, l}}{y_{k, l}}-L\right| \geq \varepsilon\right\}\right| \\
\leq \frac{1}{m n}\left|\left\{0 \leq k \leq s_{0}-1 ; 0 \leq l \leq t_{0}-1:\left|\frac{x_{k, l}}{y_{k, l}}-L\right| \geq \varepsilon\right\}\right| \\
\quad+\frac{1}{m n}\left|\left\{s_{0} \leq k \leq m-1 ; t_{0} \leq l \leq n-1:\left|\frac{x_{k, l}}{y_{k, l}}-L\right| \geq \varepsilon\right\}\right| \\
\leq \frac{M}{m n}+\frac{1}{m n}\left|\left\{s_{0} \leq k \leq m-1 ; t_{0} \leq l \leq n-1:\left|\frac{x_{k, l}}{y_{k, l}}-L\right| \geq \varepsilon\right\}\right| \leq \frac{M}{m n}+\frac{\delta}{2} .
\end{aligned}
$$

Thus, for sufficiently large $n$,

$$
\frac{1}{m n}\left|\left\{s_{0} \leq k \leq m-1 ; t_{0} \leq l \leq n-1:\left|\frac{x_{k, l}}{y_{k, l}}-L\right| \geq \varepsilon\right\}\right| \leq \frac{M}{m n}+\frac{\delta}{2}<\delta .
$$

This established the result.

Theorem 3.4 Let $\mathcal{I} \subset P(\mathbb{N} \times \mathbb{N})$ be a non-trivial ideal. Let $\bar{\theta}=\left(k_{r}, l_{s}\right)$ be a double lacunary sequence with $\liminf _{r, s} q_{r, s}>1$. Then $\left(x_{k, l}\right) \sim \mathcal{I}(\mathcal{S})^{L}\left(y_{k, l}\right) \Rightarrow\left(x_{k, l}\right) \sim \mathcal{I}\left(\mathcal{S}_{\bar{\theta}}\right)^{L}\left(y_{k, l}\right)$.

Proof Suppose that $\liminf _{r, s} q_{r, s}>1$, then there exists an $\alpha>0$ such that $q_{r, s} \geq 1+\alpha$ for sufficiently large $r, s$. Then we have

$$
\frac{h_{r, s}}{k_{r} l_{s}} \geq \frac{\alpha}{1+\alpha}
$$

If $\left(x_{k, l}\right) \sim \mathcal{I}\left(\mathcal{S}^{L}\right)\left(y_{k, l}\right)$, then for every $\varepsilon>0$ and for sufficiently large $r, s$, we have

$$
\begin{aligned}
& \frac{1}{k_{r} l_{s}}\left|\left\{k \leq k_{r} ; l \leq l_{s}:\left|\frac{x_{k, l}}{y_{k, l}}-L\right| \geq \varepsilon\right\}\right| \\
& \geq \frac{1}{k_{r} l_{s}}\left|\left\{(k, l) \in J_{r, s}:\left|\frac{x_{k, l}}{y_{k, l}}-L\right| \geq \varepsilon\right\}\right| \\
& \geq \frac{\alpha}{1+\alpha} \cdot \frac{1}{h_{r, s}}\left|\left\{(k, l) \in J_{r, s}:\left|\frac{x_{k, l}}{y_{k, l}}-L\right| \geq \varepsilon\right\}\right| .
\end{aligned}
$$

Therefore, for any $\delta>0$, we have

$$
\begin{aligned}
& \left\{(r, s) \in \mathbb{N} \times \mathbb{N}: \frac{1}{h_{r, s}}\left|\left\{(k, l) \in J_{r, s}:\left|\frac{x_{k, l}}{y_{k, l}}-L\right| \geq \varepsilon\right\}\right| \geq \delta\right\} \\
& \quad \subseteq\left\{(r, s) \in \mathbb{N} \times \mathbb{N}: \frac{1}{k_{r} l_{s}}\left|\left\{k \leq k_{r} ; l \leq l_{s}:\left|\frac{x_{k, l}}{y_{k, l}}-L\right| \geq \varepsilon\right\}\right| \geq \frac{\alpha \delta}{1+\alpha}\right\} \in \mathcal{I} .
\end{aligned}
$$

This completes the proof.

Theorem 3.5 Let $\mathcal{I}=\mathcal{I}_{\text {fin }}=\{A \subset \mathbb{N} \times \mathbb{N}: A$ is a finite set $\}$ be a non-trivial ideal, and let $\bar{\theta}=\left(k_{r}, l_{s}\right)$ be a double lacunary sequence with $\limsup _{r, s} q_{r, s}<\infty$. Then $\left(x_{k, l}\right) \sim \mathcal{I}\left(\mathcal{S}_{\bar{\theta}}\right)^{L}\left(y_{k, l}\right) \Rightarrow$ $\left(x_{k, l}\right) \sim \mathcal{I}(\mathcal{S})^{L}\left(y_{k, l}\right)$. 
Proof If $\lim \sup _{r, s} q_{r, s}<\infty$. Then there exists a $K>0$ such that $q_{r, s}<K$ for all $r, s \geq 1$. Let $\left(x_{k, l}\right) \sim \mathcal{I}\left(\mathcal{S}_{\bar{\theta}}\right)^{L}\left(y_{k, l}\right)$. Then there exists $B>0$ and $\varepsilon>0$, we put

$$
M_{r, s}=\frac{1}{h_{r, s}}\left|\left\{(k, l) \in J_{r, s}:\left|\frac{x_{k, l}}{y_{k, l}}-L\right| \geq \varepsilon\right\}\right| .
$$

Since $\left(x_{k, l}\right) \sim^{\mathcal{I}\left(\mathcal{S}_{\bar{\theta}}\right)^{L}}\left(y_{k, l}\right)$. Then for every $\varepsilon>0$ and $\delta>0$, we have

$$
\begin{aligned}
& \left\{(r, s) \in \mathbb{N} \times \mathbb{N}: \frac{1}{h_{r, s}}\left|\left\{(k, l) \in J_{r, s}:\left|\frac{x_{k, l}}{y_{k, l}}-L\right| \geq \varepsilon\right\}\right| \geq \delta\right\} \\
& \quad=\left\{(r, s) \in \mathbb{N} \times \mathbb{N}: \frac{M_{r, s}}{h_{r, s}} \geq \delta\right\} \in \mathcal{I},
\end{aligned}
$$

and, therefore, it is a finite set. We choose integers $r_{0}, s_{0} \in \mathbb{N}$ such that

$$
\frac{M_{r, s}}{h_{r, s}}<\delta \quad \text { for all } r>r_{0}, s>s_{0}
$$

Let $M=\max \left\{M_{r, s}: 1 \leq r \leq r_{0}, 1 \leq s \leq s_{0}\right\}$ and $m, n$ be two integers with satisfying $k_{r-1}<$ $m \leq k_{r}, l_{s-1}<n \leq l_{s}$, then we have

$$
\begin{aligned}
& \frac{1}{m n}\left|\left\{k \leq m ; l \leq n:\left|\frac{x_{k, l}}{y_{k, l}}-L\right| \geq \varepsilon\right\}\right| \\
& \quad \leq \frac{1}{k_{r-1} l_{s-1}}\left|\left\{k \leq k_{r} ; l \leq l_{s}:\left|\frac{x_{k, l}}{y_{k, l}}-L\right| \geq \varepsilon\right\}\right| \\
& =\frac{1}{k_{r-1} l_{s-1}}\left\{M_{1,1}+M_{2,2}+\cdots+M_{r_{0}, s_{0}}+M_{r_{r_{0}+1, s_{0}+1}}+\cdots+M_{r, s}\right\} \\
& \leq \frac{M}{k_{r-1} l_{s-1}} \cdot r_{0} s_{0}+\frac{1}{k_{r-1} l_{s-1}}\left\{h_{r_{0}+1, s_{0}+1}\left(\frac{M_{r_{0}+1, s_{0}+1}}{h_{r_{0}+1, s_{0}+1}}\right)+\cdots+h_{r, s} \frac{M_{r, s}}{k_{r, s}}\right\} \\
& \leq \frac{M}{k_{r-1} l_{s-1}} \cdot r_{0} s_{0}+\frac{1}{k_{r-1} l_{s-1}}\left(\sup _{r>r_{0}, s>s_{0}} \frac{M_{r, s}}{h_{r, s}}\right)\left\{h_{r_{0}+1, s_{0}+1}+\cdots+h_{r, s}\right\} \\
& \leq \frac{M}{k_{r-1} l_{s-1}} \cdot r_{0} s_{0}+\delta\left(\frac{k_{r} l_{s}-k_{r_{0}} l_{s_{0}}}{k_{r-1} l_{s-1}}\right) \\
& \leq \frac{M}{k_{r-1} l_{s-1}} \cdot r_{0} s_{0}+\delta \cdot q_{r, s} \leq \frac{M}{k_{r-1} l_{s-1}} \cdot r_{0} s_{0}+\delta K .
\end{aligned}
$$

This completes the proof of the theorem.

Definition 3.13 Let $p \in(0, \infty)$. Two non-negative double sequences $\left(x_{k, l}\right)$ and $\left(y_{k, l}\right)$ are said to be strongly asymptotically lacunary p-equivalent of multiple $L$ if

$$
P-\lim _{r, s} \frac{1}{h_{r, s}} \sum_{(k, l) \in J_{r, s}}\left|\frac{x_{k, l}}{y_{k, l}}-L\right|^{p}=0
$$

denoted by $\left.\left(x_{k, l}\right) \sim{ }^{\left[N_{\bar{\theta}}\right.}\right]_{p}^{L}\left(y_{k, l}\right)$ and simply strongly asymptotically lacunary $p$-equivalent if $L=1$. 
Definition 3.14 Let $p \in(0, \infty)$. Two non-negative double sequences $\left(x_{k, l}\right)$ and $\left(y_{k, l}\right)$ are said to be asymptotically lacunary $p$-statistically equivalent of multiple $L$ if for every $\varepsilon>0$,

$$
P-\lim _{r, s} \frac{1}{h_{r, s}}\left|\left\{(k, l) \in J_{r, s}:\left|\frac{x_{k, l}}{y_{k, l}}-L\right|^{p} \geq \varepsilon\right\}\right|=0
$$

denoted by $\left(x_{k, l}\right) \sim \mathcal{S}_{\overline{\theta_{p}}}^{L}\left(y_{k, l}\right)$ and simply asymptotically lacunary $p$-statistical equivalent if $L=1$.

Theorem 3.6 Let $\bar{\theta}=\left(k_{r}, l_{s}\right)$ be a double lacunary sequence. Then

(a) $\left(x_{k, l}\right) \sim^{\left[N_{\bar{\theta}}\right]_{p}^{L}}\left(y_{k, l}\right) \Rightarrow\left(x_{k, l}\right) \sim^{\mathcal{S}_{\bar{\theta}}^{L}}\left(y_{k, l}\right)$.

(b) $\left[N_{\bar{\theta}}\right]_{p}^{L}$ is a proper subset of $\mathcal{S}_{\bar{\theta}_{p}}^{L}$.

(c) Let $\left(x_{k, l}\right),\left(y_{k, l}\right) \in \ell_{\infty}^{2}$ and $\left(x_{k m l}\right) \sim^{\mathcal{S}_{\bar{\theta}}^{L}}\left(y_{k, l}\right)$, then $\left(x_{k, l}\right) \sim^{\left[N_{\bar{\theta}}\right]_{p}^{L}}\left(y_{k, l}\right)$.

(d) $\mathcal{S}_{\bar{\theta}_{p}}^{L} \cap \ell_{\infty}^{2}=\left[N_{\bar{\theta}}\right]_{p}^{L} \cap \ell_{\infty}^{2}$.

The proof of the above theorem is similar to Theorem 3.2 for $I=I_{\text {fin }}$.

Definition 3.15 Let $p \in(0, \infty)$. We say that two non-negative double sequences $\left(x_{k, l}\right)$ and $\left(y_{k, l}\right)$ are strongly asymptotically $\mathcal{I}$-lacunary p-equivalent of multiple $L$ if for every $\varepsilon>0$,

$$
\left\{(r, s) \in \mathbb{N} \times \mathbb{N}: \frac{1}{h_{r, s}} \sum_{(k, l) \in J_{r, s}}\left|\frac{x_{k, l}}{y_{k, l}}-L\right|^{p} \geq \varepsilon\right\} \in \mathcal{I}
$$

denoted by $\left(x_{k, l}\right) \sim^{\mathcal{I}\left(N_{\bar{\theta}}\right)_{p}^{L}}\left(y_{k, l}\right)$ and simply strongly asymptotically $\mathcal{I}$-lacunary $p$-equivalent if $L=1$.

Definition 3.16 Let $p \in(0, \infty)$. We say that two non-negative double sequences $\left(x_{k, l}\right)$ and $\left(y_{k, l}\right)$ are asymptotically $\mathcal{I}$-lacunary $p$-statistically equivalent of multiple $L$ if for every $\varepsilon>0$, for every $\delta>0$

$$
\left\{(r, s) \in \mathbb{N} \times \mathbb{N}: \frac{1}{h_{r, s}}\left|\left\{(k, l) \in J_{r, s}:\left|\frac{x_{k, l}}{y_{k, l}}-L\right|^{p} \geq \varepsilon\right\}\right| \geq \delta\right\} \in \mathcal{I}
$$

denoted by $\left(x_{k, l}\right) \sim^{\mathcal{I}\left(\mathcal{S}_{\bar{\theta}}\right)^{L}}\left(y_{k, l}\right)$ and simply asymptotically $\mathcal{I}$-lacunary $p$-statistical equivalent if $L=1$.

Theorem 3.7 Let $\mathcal{I} \subset P(\mathbb{N} \times \mathbb{N})$ be a non-trivial ideal, and let $\bar{\theta}=\left(k_{r}, l_{s}\right)$ be a double lacunary sequence. Then
(a) $\left(x_{k, l}\right) \sim^{\mathcal{I}\left(N_{\bar{\theta}_{p}}\right)^{L}}\left(y_{k, l}\right) \Rightarrow\left(x_{k, l}\right) \sim^{\mathcal{I}\left(\mathcal{S}_{\bar{\theta}_{p}}\right)^{L}}\left(y_{k, l}\right)$.
(b) $\mathcal{I}\left(N_{\bar{\theta}_{p}}\right)^{L}$ is a proper subset of $\mathcal{I}\left(\mathcal{S}_{\bar{\theta}_{p}}\right)^{L}$.
(c) Let $\left(x_{k, l}\right),\left(y_{k, l}\right) \in \ell_{\infty}^{2}$ and $\left(x_{k, l}\right) \sim^{\mathcal{I}\left(\mathcal{S}_{\bar{\theta}_{p}}\right)^{L}}\left(y_{k, l}\right)$, then $\left(x_{k}\right) \sim^{I\left(N_{\theta_{p}}\right)^{L}}\left(y_{k}\right)$.
(d) $\mathcal{I}\left(\mathcal{S}_{\bar{\theta}_{p}}\right)^{L} \cap \ell_{\infty}^{2}=\mathcal{I}\left(N_{\bar{\theta}_{p}}\right)^{L} \cap \ell_{\infty}^{2}$.

Proof The proof of the theorem follows from the proofs of the Theorems 3.2 and 3.6.

For $\mathcal{I}=\mathcal{I}_{\text {fin }}=\{A \subseteq \mathbb{N} \times \mathbb{N}: A$ is finite $\}$, this theorem reduces to Theorem 3.6. 


\section{Competing interests}

The authors declare that they have no competing interests.

\section{Authors' contributions}

BH drafted the manuscript. VK checked and organized the manuscript to be its final form. BH also makes the revision as the corresponding author.

\section{Author details}

'Department of Mathematics, Rajiv Gandhi University, Rono Hills, Doimukh, Arunachal Pradesh 791112, India.

${ }^{2}$ Department of Mathematics, Haryana College of Technology and Management, Kaithal, Haryana 136027, India.

\section{Acknowledgements}

The authors thank all three reviewers for their useful comments that led to the improvement of the original manuscript.

Received: 3 April 2013 Accepted: 24 September 2013 Published: 19 Nov 2013

\section{References}

1. Pobyvancts, IP: Asymptotic equivalence of some linear transformation defined by a nonnegative matrix and reduced to generalized equivalence in the sense of Cesaro and Abel. Mat. Fiz. 28, $83-87$ (1980)

2. Marouf, MS: Asymptotic equivalence and summability. Int. J. Math. Math. Sci. 16(4), 755-762 (1993)

3. Patterson, RF: On asymptotically statistically equivalent sequences. Demonstr. Math. 36(1), 149-153 (2003)

4. Patterson, RF, Savaş, E: On asymptotically lacunary statistically equivalent sequences. Thai J. Math. 4, 267-272 (2006)

5. Zygmund, A: Trigonometric Series. Cambridge University Press, Cambridge (1979)

6. Steinhaus, H: Sur la convergence ordinate et la convergence asymptotique. Colloq. Math. 2, $73-84$ (1951)

7. Fast, H: Sur la convergence statistique. Colloq. Math. 2, 241-244 (1951)

8. Schoenberg, IJ: The integrability of certain functions and related summability methods. Am. Math. Monthly 66, 361-375 (1959)

9. Buck, RC: Generalized asymptotic density. Am. J. Math. 75, 335-346 (1953)

10. Šalát, T: On statistical convergence of real numbers. Math. Slovaca 30, 139-150 (1980)

11. Fridy, JA: On statistical convergence. Analysis 5, 301-313 (1985)

12. Fridy, JA, Orhan, C: Lacunary statistical convergence. Pac. J. Math. 160(1), 43-51 (1993)

13. Mursaleen, M, Mohiuddine, SA: On lacunary statistical convergence with respect to the intuitionistic fuzzy normed space. J. Comput. Appl. Math. 233(2), 142-149 (2009)

14. Savaş, E, Patterson, RF: Lacunary statistical convergence of double sequences. Math. Commun. 10, 55-61 (2005)

15. Savaş, E, Patterson, RF: Lacunary statistical convergence of multiple sequences. Appl. Math. Lett. 19, 527-534 (2006)

16. Mohiuddine, SA, Alotaibi, A, Mursaleen, M: Statistical convergence of double sequences in locally solid Riesz spaces. Abstr. Appl. Anal. 2012, Article ID 719729 (2012)

17. Çakalli, H: Lacunary statistical convergence in topological groups. Indian J. Pure Appl. Math. 26(2), 113-119 (1995)

18. Çakan, C, Altay, B, Çoşkun, H: Double lacunary density and lacunary statistical convergence of double sequences. Studia Sci. Math. Hung. 47(1), 35-45 (2010)

19. Fridy, JA, Orhan, C: Lacunary statistical summability. J. Math. Anal. Appl. 173, 497-504 (1993)

20. Hazarika, B, Savaş, E: Lacunary statistical convergence of double sequences and some inclusion results in $n$-normed spaces. Acta Math. Vietnam. (2013). doi:10.1007/s40306-013-0028-x

21. Li, J: Lacunary statistical convergence and inclusion properties between lacunary methods. Int. J. Math. Math. Sci. 23(3), 175-180 (2000)

22. Mohiuddine, SA, Savas, E: Lacunary statistical convergent double sequences in probabilistic normed spaces. Ann. Univ. Ferrara 58, 331-339 (2012). doi:10.1007/s11565-012-0157-5

23. Mohiuddine, SA, Aiyub, M: Lacunary statistical convergence in random 2-normed spaces. Appl. Math. Inform. Sci. 6(3), 581-585 (2012)

24. Savas, E: Double lacunary statistically convergent sequences in topological groups. J. Franklin Inst. (in press). doi:10.1016/j.jfranklin.2012.06.003

25. Kostyrko, P, Salát, T, Wilczyński, W: I-convergence. Real Anal. Exch. 26(2), 669-686 (2000-2001)

26. Das, P, Savas, E, Ghosal, S: On generalization of certain summability methods using ideal. Appl. Math. Lett. 24 1509-1514 (2011)

27. Choudhary, B: Lacunary I-convergent sequences. In: Real Analysis Exchange Summer Symposium, pp. 56-57 (2009)

28. Tripathy, BC, Hazarika, B, Choudhary, B: Lacunary I-convergent sequences. Kyungpook Math. J. 52(4), 473-482 (2012)

29. Hazarika, B: Lacunary I-convergent sequence of fuzzy real numbers. Pac. J. Sci. Technol. 10(2), $203-206$ (2009)

30. Hazarika, B: Fuzzy real valued lacunary /-convergent sequences. Appl. Math. Lett. 25(3), 466-470 (2012)

31. Kumar, V, Sharma, A: Asymptotically lacunary equivalent sequences defined by ideals and modulus function. Math. Sci. 6, 23 (2012). doi:10.1186/2251-7456-6-23

32. Savas, E: On $\mathcal{I}$-asymptotically lacunary statistical equivalent sequences. Adv. Differ. Equ. 2013, 111 (2013) doi:10:1186/1687-1847-2013-111

33. Savas, E, Gumus, H: A generalization on $\mathcal{I}$-asymptotically lacunary statistical equivalent sequences. J. Inequal. Appl. 2013, $436(2013)$

34. Çakalli, H, Hazarika, B: Ideal quasi-Cauchy sequences. J. Inequal. Appl. 2012, 234 (2012). doi:10.1186/1029-242X-2012-234

35. Dutta, AJ, Tripathy, BC: On I-acceleration convergence of sequences of fuzzy real numbers. Math. Model. Anal. 17(4), 549-557 (2012)

36. Esi, A, Hazarika, B: Lacunary summable sequence spaces of fuzzy numbers defined by ideal convergence and an Orlicz function. Afr. Math. (2012). doi:10.1007/s13370-012-0117-3

37. Hazarika, B: On ideal convergence in topological groups. Sci. Magna 7(4), 80-86 (2011)

38. Hazarika, B: Lacunary difference ideal convergent sequence spaces of fuzzy numbers. J. Intell. Fuzzy Syst. 25(1), 157-166 (2013). doi:10.3233/IFS-2012-0622 
39. Lahiri, BK, Das, P: / and /*-convergence in topological spaces. Math. Bohem. 130(2), 153-160 (2005)

40. Mohiuddine, SA, Alotaibi, A, Alsulami, SM: Ideal convergence of double sequences in random 2-normed spaces. Adv. Differ. Equ. 2012, 149 (2012)

41. Šalát, T, Tripathy, BC, Ziman, M: On some properties of /-convergence. Tatra Mt. Math. Publ. 28, $279-286$ (2004)

42. Šalát, T, Tripathy, BC, Ziman, M: On I-convergence field. Indian J. Pure Appl. Math. 17, 45-54 (2005)

43. Tripathy, BC, Hazarika, B: Paranorm /-convergent sequence spaces. Math. Slovaca 59(4), 485-494 (2009)

44. Tripathy, BC, Hazarika, B: I-monotonic and I-convergent sequences. Kyungpook Math. J. 51, 233-239 (2011). doi:10.5666/KMJ.2011.51.2.233

45. Tripathy, BC, Hazarika, B: I-convergent sequence spaces associated with multiplier sequences. Math. Inequal. Appl. 11(3), 543-548 (2008)

46. Tripathy, BC, Sen, M, Nath, S: I-convergence in probabilistic n-normed space. Soft Comput. 16, 1021-1027 (2012)

47. Tripathy, BC, Mahanta, S: On I-acceleration convergence of sequences. J. Franklin Inst. 347, 1031-1037 (2010)

48. Freedman, AR, Sember, JJ, Raphael, M: Some Cesàro-type summability spaces. Proc. Lond. Math. Soc. 37(3), 508-520 (1978)

49. Pringsheim, A: Zur theorie der zweifach unendlichen zahlenfolgen. Math. Ann. 53, 289-321 (1900)

50. Mursaleen, M, Edely, OHH: Statistical convergence of double sequences. J. Math. Anal. Appl. 288, $223-231$ (2003)

51. Tripathy, BK, Tripathy, BC: On I-convergent double sequences. Soochow J. Math. 31(4), 549-560 (2005)

10.1186/1029-242X-2013-543

Cite this article as: Hazarika and Kumar: On asymptotically double lacunary statistical equivalent sequences in ideal context. Journal of Inequalities and Applications 2013, 2013:543

\section{Submit your manuscript to a SpringerOpen ${ }^{\circ}$ journal and benefit from:}

- Convenient online submission

- Rigorous peer review

- Immediate publication on acceptance

- Open access: articles freely available online

- High visibility within the field

- Retaining the copyright to your article 